\title{
Electron Spins Are Key in Nickelate Superconductors
}

\author{
A newly discovered superconductor has a surprisingly low transition \\ temperature, but its electronic structure points the way to materials that \\ superconduct at higher temperatures.
}

By Sophia Chen

n 2019, physicists reported the discovery

of the first nickel-based superconductor, $(\mathrm{Nd}, \mathrm{Sr}) \mathrm{NiO}_{2}$.

The material's electronic structure resembles the famous copper-oxide-based (cuprate) superconductors. But while the superconducting transition temperature for cuprates can be as high as $165 \mathrm{~K},(\mathrm{Nd}, \mathrm{Sr}) \mathrm{NiO}_{2}$ must be cooled below $15 \mathrm{~K}$ to become superconducting. In new theoretical work, Hirofumi Sakakibara, of Tottori University in Japan, and colleagues develop a model of the new nickelate superconductor that explains its surprisingly low transition temperature [1]. By comparing the superconducting mechanisms of nickelates and cuprates, the researchers hope to better understand the phenomena that produce superconductivity at higher temperatures.

For a material to be superconducting, the outermost electrons in its atoms must overcome their mutual repulsion to link up in so-called Cooper pairs. In cuprate superconductors, this

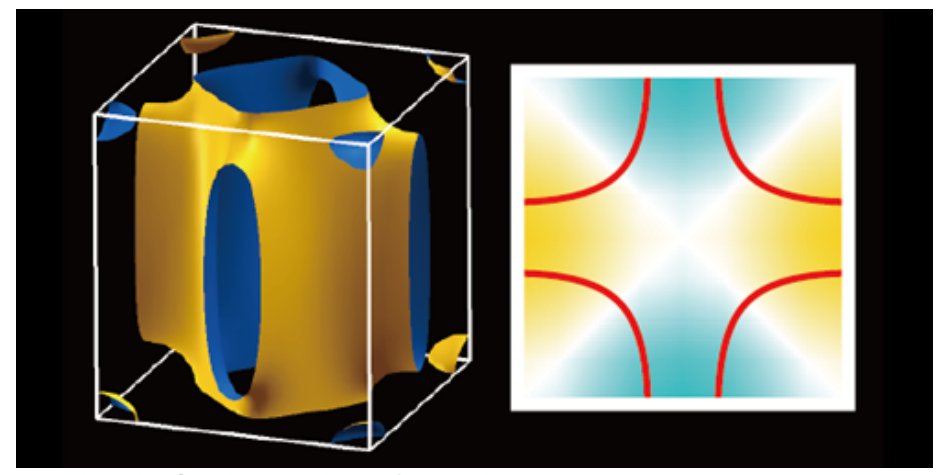

Credit: Hirofumi Sakakibara/Tottori University process is promoted by the fluctuations of electron spins. The spins trigger a collective motion that pushes some electrons together, enabling Cooper-pair formation.

To see whether the same mechanism governs superconductivity in nickelates, Sakakibara and colleagues modeled the interaction of electrons in ( $\mathrm{Nd}, \mathrm{Sr}) \mathrm{NiO}_{2}$ using first principles calculations. The researchers found that, as in cuprate superconductors, Cooper pairs form in ( $\mathrm{Nd}, \mathrm{Sr}) \mathrm{NiO}_{2}$ because fluctuating electron spins force electrons together. But they also found that electrons in the nickelate interact more strongly than electrons in cuprates. These strong interactions reduce the superconducting temperature by inhibiting charges from moving through the material. The team's results suggest that one strategy for discovering additional high-temperature superconductors such as cuprates is to investigate materials in which these electron interactions are weaker, for example nickelates under high pressure.

This research is published in Physical Review Letters.

Sophia Chen is a freelance science writer based in Columbus, Ohio.

\section{REFERENCES}

1. H. Sakakibara et al., "Model construction and a possibility of cupratelike pairing in a new $d^{9}$ nickelate superconductor (Nd,Sr) $\mathrm{NiO}_{2}$," Physical Review Letters 125, 077003 (2020). 